\title{
THE COSMIC NOZZLE
}

\section{KONJUKOV M.V.}

Astrospace Center of P.N.Lebedev Physics Institute of RAS Moscow Leninskii prospekt 53 Russia

The cosmic nozzle is a channel with flowing hot gas in a system containing the following components: stars, a hydrostatic gaseous corona and a cavity with hot gas in the central part of the system. In the quasi onedimension approximation, the following solution of the cosmic nozzle problem about cosmic nozzle was obtained

$$
\begin{gathered}
\nu=\nu_{0} \pi_{g}^{3 / 5}, \quad w=\sqrt{5 \tau_{0}}\left[1-\pi_{g}^{2 / 5}-\frac{2}{5 \tau_{0}}\left(\psi_{0}-\psi\right)+\frac{w_{0}^{2}}{5 \tau_{0}}\right]^{1 / 2} \\
\tau=\tau_{0} \pi_{g}^{2 / 5}, \quad \sigma=\sigma_{0} \frac{w_{0} / \sqrt{5 \tau_{0}}}{\pi_{g}^{3 / 5}\left[1-\pi_{g}^{2 / 5}-\frac{2}{5 \tau_{0}}\left(\psi_{0}-\psi\right)+\frac{w_{0}^{2}}{5 \tau_{0}}\right]^{1 / 2}} .
\end{gathered}
$$

Here $(\nu, w, \tau, \sigma)$ are the dimensionless density, hydrodynamical velocity, temperature and cross section of the channel $\left(\nu_{0}, w_{0}, \tau_{0}, \sigma_{0}\right.$ are these values at $\xi=1), \phi(\xi), \pi_{g}(\xi)$ are the gravitational potential and the pressure in the gaseous corona. For a polytropic state equation of the gas in the gaseous corona

$$
\psi_{0}-\psi(\xi)=\frac{\kappa_{0}}{\kappa_{0}-1}\left(1-\pi_{g}^{\frac{\kappa_{0}-1}{\kappa_{0}}}\right)
$$

The investigations of the solutions yield the properties of the flows in cosmic nozzle:

1.The distributions of the density and the temperature of the outflowing hot gas depend only from the pressure in the surrounding gaseous corona $\nu=\nu_{0} \pi_{g}(\xi)^{3 / 5}, \quad \tau=\tau_{0} \pi_{g}(\xi)^{2 / 5}$. They do not depend on non hydrodynamical forces acting on flowing gas in the channel.

2.For any dimensionless values determining the properties of the cosmic nozzle there is a nonmonotonically changing velocity. It has a maximum at $\xi_{*}$ determined by the equation $\pi_{g}(\xi)=\tau_{0}{ }^{5 \kappa_{0} /\left(3 \kappa_{0}-5\right)}$. Physically this is 
the simple consequence of the transition from $\nu / \nu_{g}<1$ to $\nu / \nu_{g}>1$. That property of the velocity disappear by the neglecting of gravity forces.

3.In the broad region of the values of the parameters of the cosmic nozzle, there is nonmonotoncally change of the channel cross section, with a minimum at finite distance from the channel origin.

4.The flow in the cosmic nozzle has a narrow layer near the channel origin where the velocity changes sharply.

5.There is the following formula for the velocity in the channel

$$
u=\sqrt{\frac{5 k T_{0}^{c}}{m}}\left(1-\tau-\frac{2}{5} \frac{m}{k T_{0}^{c}}\left(\Phi_{0}-\Phi\right)+\frac{m u_{0}^{2}}{5 k T_{0}^{c}}\right)^{1 / 2}
$$

where $T_{0}^{c}$ is the gas temperature in the cavity. As long as the cavity gas is hot and the gas velocity at the channel origin is subsonic, an asymptotic series for the velocity can be obtained, with main term

$$
u_{a s}=\sqrt{\frac{5 k T_{0}^{c}}{m}}=\sqrt{\frac{5 k T_{0}^{g}}{3 m}} \sqrt{\frac{3 k T_{0}^{c}}{T_{0}^{g}}},
$$

where $\sqrt{5 k T_{0}^{g} / 3 m}$ is the adiabatic sound velocity for corona gas at the boundary with the cavity. The values of $u_{a s}$ are nonrelativistic for reasonable temperatures of the cavity and corona.

6. For rough estimations of the energy flow the formula

$$
E_{0}=\frac{5}{2} w_{0} S_{0} n_{0}^{g} T_{0}^{g} \sqrt{\frac{5 k T_{0}^{g}}{3 m}}
$$

can be used, where $w_{0}=u_{0} / \sqrt{\frac{5 k T_{0}^{c}}{3 m}}$. Even at the radian measure $S_{0} \approx 1$, $w_{0}<1$ and extreme conditions the energy flow is less then $10^{40} \mathrm{erg} / \mathrm{sec}$.

7.There are no physically plausible solutions for the gaseous corona with $\kappa_{0}$ near 1.

8.There are difficulties with the fulfillment of the hydrodynamical approximation in the channel.

9.The set of differential equations for the cosmic nozzle does not have singular points when hydrodynamical velocity equals the local sound velocity.

There are two main consequences from the above obtained results:

1.The cosmic nozzle model cannot explain the main properties of FR-II radio sources.

2.There is no small parameter of the problem for which the set of cosmic nozzle equations is an approximation to the quasi one-dimension gasdynamic equations. 